\title{
Evaporation of liquefied natural gas in conditions of compact storage containers heating
}

\author{
D.S. Telgozhayeva
}

Kazakhstan

\begin{abstract}
Identical by its power, but located in different parts of the external surface of the tank, the heating sources are different intensity heat transfer modes is heating up, respectively, times of vapour pressure rise to critical values. Developed mathematical model and method of calculation can be used in the analysis of conditions of storage tanks for liquefied gases.
\end{abstract}

\section{Introduction}

The growth of production and consumption of liquefied natural gas (LNG) leads to the development of special measures for the safe storage and use of CNG [1]. One of the challenges when storing such gases is to reduce losses from the main product from the "evaporation" [1]. This task cannot only empirically. The most promising is the development of methods of calculation of parameters of heat transfer in evaporation of LNG in large tanks at long storage, and in small containers at a local intensive heating.

The purpose of this work is the mathematical modeling of heat transfer in partially filled with smallsize evaporation capacity liquefied natural gas under intense local heating.

When there are two approaches, tasks related to the conditions of supply of heat to certain sections of the external surface of the tank. Local heating on the lateral surface, the field of analysis, heat transfer will be carried out through natural convection and heat conduction. If the heating zone is located below the coordinates $\mathrm{Z}=\mathrm{Z} 1$ (Fig. 1), then warm the LNG will be sufficiently utilized due to the relatively high thermal conductivity LNG (versus his pairs). While there are processes and convective conductive. Because of the uneven heating of the liquefied natural gas in $0 \leq \mathrm{Z} \leq \mathrm{Z} 1$, will be free convective currents. As a result of the warming up of CNG will proceed more intensively than when transferring heat conduction only.

If the local source of heating is located in the $\mathrm{Z} 1 \leq \mathrm{Z} \leq \mathrm{Z} 2$, the convective processes will occur in the vapor of LNG. Accordingly, due to the smaller, compared with liquefied gas thermal conductivity, the intensity of a conductive heating of vapour phase will be smaller than the first version.

If the tank is heated by a top end surface (Fig. 1), then the dominant is heat transfer by conduction in the vapour phase. Accordingly, the rate of temperature rise at the interface «pairs - LNG» is the smallest of the three discussed possible options for local heating of the tank with LNG. This paper discusses the option of heating from the top of the analysis, as one of the most likely in practice.

This is an Open Access article distributed under the terms of the Creative Commons Attribution License 4.0, which permits unrestricted use, distribution, and reproduction in any medium, provided the original work is properly cited. 


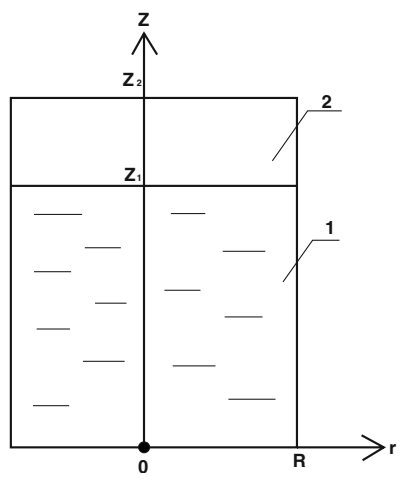

Figure 1. Scope of the challenge: 1 - liquefied gas; 2 - gas in the gaseous state.

\section{Problem statement}

The problem of heat conduction in two-layer floor cylinder (Fig. 1). Most of the internal volume of the cylinder is CNG. About $15 \%$ of the volume is occupied by natural gas in the gaseous state. The challenge is in a 2D setting in the cylindrical coordinate system. In actual practice, there is a small tank with LNG heat only on a small area of its external surface. Therefore, the three outer edges are various boundary conditions. The lower surface of the cylinder is properly insulated. On the side (problem is solved in axisymmetric formulation) set the boundary conditions of the first kind (the constant low temperature). At the top of the cooking conditions are simulated (set temperature, greatly exceeding the initial temperature of the decision). The fourth kind boundary conditions are defined on the boundary of phases. In this condition is the heat of phase transition (evaporation of LNG). Massive evaporation rate was calculated using a mathematical expression of the Hertz-Knudsen, widely used in solving problems of heat and mass transfer in conditions of intensive evaporation of liquids [2, 3]. Vaporization in the area (2) (Fig. 1) was seen as a process, as a result of which rises the pressure in the tank. Natural gas was considered a heat-shrinkable liquid. LNG vaporization products pressure calculated using the ideal gas equation.

\section{Mathematical model}

Mathematical model of the process is as follows:

$$
\begin{gathered}
C_{1} \rho_{1} \frac{\partial T_{1}}{\partial t}=\lambda_{1}\left[\frac{1}{r} \frac{\partial}{\partial r}\left(r \frac{\partial T_{1}}{\partial r}\right)+\frac{\partial^{2} T_{1}}{\partial Z^{2}}\right], \\
C_{2} \rho_{2} \frac{\partial T_{2}}{\partial t}=\lambda_{2}\left[\frac{1}{r} \frac{\partial}{\partial r}\left(r \frac{\partial T_{2}}{\partial r}\right)+\frac{\partial^{2} T_{2}}{\partial Z^{2}}\right], \\
T_{1}=T_{0} \quad T_{2}=T_{0} \quad t=0 ; \\
\frac{\partial T_{1}}{\partial r}=0 \quad r=0,0<Z<Z_{1} ;
\end{gathered}
$$




$$
\begin{gathered}
T_{1}=T_{k} \quad r=R, 0<Z<Z_{1} ; \\
\frac{\partial T_{1}}{\partial Z}=0 \quad Z=0,0<r<R \\
\frac{\partial T_{2}}{\partial r}=0 \quad r=0, Z_{1}<Z<Z_{2} ; \\
T_{2}=T_{k} \quad r=R, Z_{i}<Z<Z_{2} ; \\
T_{2}=T_{\mathrm{H}} \quad Z=Z_{2}, 0<r<R ; \\
\left.-\lambda_{1} \frac{\partial T_{1}}{\partial Z}=-\lambda_{2} \frac{\partial T_{2}}{\partial Z}+Q . W\right\} Z=Z_{1}, 0<r<R . \\
\beta \quad \frac{\left(P^{\mathrm{H}}-P\right)}{\sqrt{2 \pi R T / M} ; P=\frac{\rho R_{2} T}{M} .}
\end{gathered}
$$

\section{Results of numerical simulation}

Heat transfer solved task formulated by finite differences method using iterative algorithm [4], developed in heat and mass transfer in the ignition of flammable liquids at an intensive evaporation of the past.

Due to intense absorption of heat in the narrow area of the phase transition on the boundary of environments and, accordingly, the emergence of large temperature gradients was uneven and irregular differential grid. To ensure sustainability and convergence of numerical solutions to the time selected in the range of 10-2 to 10-6 c, similar to the algorithm [5, 6].

In Fig. 2 are the typical distribution of temperature in $\mathrm{Z}$ section $r=0$ on the axis of symmetry of the cylinders at different points in time, the numerical solution of systems of equations is formulated with relevant regional conditions.

Analysis of the numerical simulation showed that, even at relatively short rise in temperature at one of the boundaries of the region as a result of intensive evaporation of LPG vapour pressure would grow to values significantly in excess of the permissible (Fig. 3). Rapid rise in pressure in the case of heating of the upper border of the area where the gas is due to the exponential dependence of the evaporation rate of LNG from the temperature.

Analysis of the figure according to the conclusion and substantially changing the non $\mathrm{P}$ with increasing time. Curve $\mathrm{P}(\mathrm{t})$ due to the specific processes of heat transfer in tanks for storage liquefied natural gas when heated at the top. In this case, the mode is not implemented by natural convection and heat conduction only. Due to the low thermal conductivity of vapor of LNG in critical temperatures warming up as vapour, and then liquefied gas going rather slowly. As a result the temperature boundary section "liquefied gas-couples" is only at time intervals more than $150 \mathrm{~s}$ (for the heating conditions and the size of the area). The increased thickness of the steam layer in the initial time is increased and the 


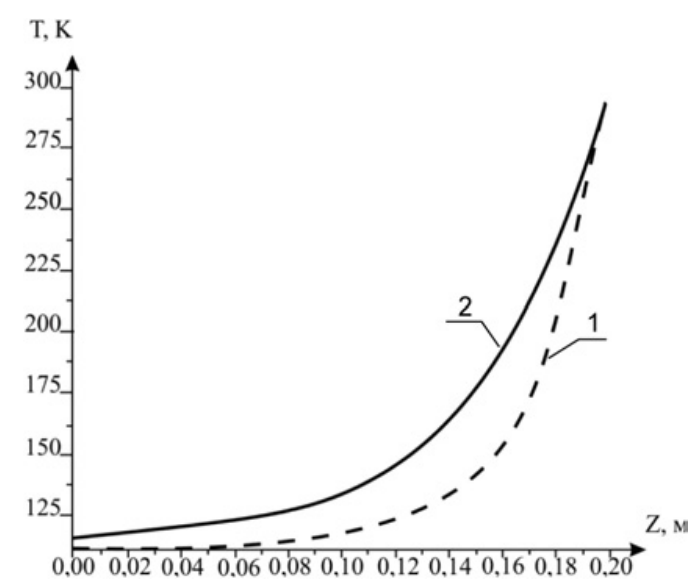

Figure 2. The height of the cylinder temperature distribution at different points in time.

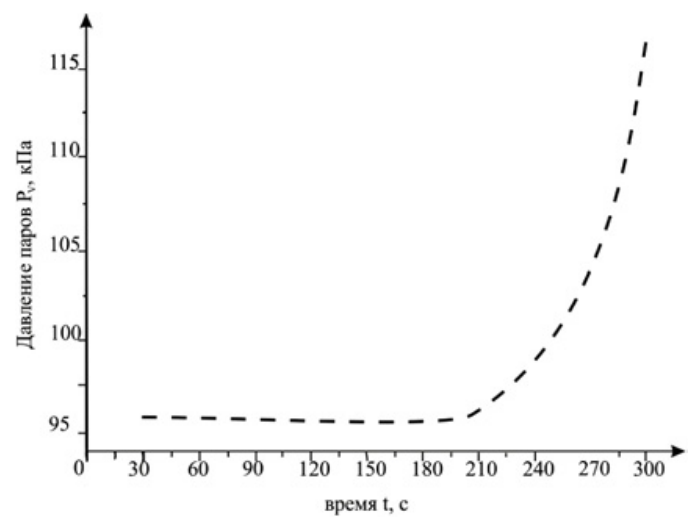

Figure 3. Change of the vapour pressure of the LNG in time.

time it was heating up. Accordingly, the reduction of this thickness leads to faster recovery of pressure in the tank.

The results also show that the heat capacity of the lateral border (as in the interval corresponding to the zone of vapour and LNG) most likely will change the real mechanism of heat transfer in vapor. Local heating of the side surface portion and the corresponding temperature increase of the tank inner surface will lead to the formation of free-convective flow in small-size region near the interface «couples - LNG». With the growth of time, the process of natural convection will be intensified and include more and more filled with steam space. In this case, the heat transfer to the surface of the LNG will be better (than the thermal conductivity only) speed. Accordingly, the vapour pressure rise time is reduced to a critical (the destruction of the tank) pressure.

\section{Conclusion}

It can be concluded that when identical by its power, but located in different parts of the external surface of the tank, the heating sources are different intensity heat transfer modes and LNG is heating up, respectively, times of vapour pressure rise to critical values. 
Developed mathematical model and method of calculation can be used in the analysis of conditions of storage tanks for liquefied gases.

\section{Notations}

$T$ - temperature; $C$ - heat capacity; $\lambda$ - thermal conductivity; $\rho$ - density; $r, Z$ - coordinates the cylindrical coordinate system; $T_{k}$ - the temperature of the outer border; $T_{\mathrm{H}}$ - temperature of the heat source; $Q, W$ - heat effect and massive evaporation rate of LNG, accordingly; $\beta$ - dimensionless coefficient of vaporization; $k$ - constant; $P^{\mathrm{H}}$ saturated vapor pressure of gas; $P$ - the vapour pressure of gas near the border of evaporation; $M$ - the molecular weight of natural gas; $R_{2}$ gas constant; $R, Z_{2}-$ coordinates of the outer limit of; $Z_{1}$ - coordinate border section «liquid - gas», index 1 corresponds to liquefied gas, 2 - gas in the gaseous state.

\section{References}

[1] Rachevsky B.S. Liquefied petroleum gases. - M..: 2009. - 639c

[2] Kuznetsov G.V., Strizhak P.A. The influence of heat transfer conditions at the hot particle-liquid fuel interface on the ignition characteristics// Journal of Engineering Thermophysics. 2009. T. 18. No. 2. C. $162-167$

[3] Kuznetsov G.V., Strizhak P.A. 3D problem of heat and mass transfer at the ignition of a combustible liquid by a heated metal particle// Journal of Engineering Thermophysics. 2009. T. 18. No. 1. C. 72-79

[4] Kuznetsov G.V., Strizhak P.A. Transient heat and mass transfer at the ignition of vapor and gas mixture by a moving hot particle// International Journal of Heat and Mass Transfer. 2010. T. 53. No. 5-6. C. 923-930

[5] Strakhov V.L., Garashchenko A.N., Kuznetsov G.V., Rudzinskii V.P. Mathematical simulation of thermophysical and thermochemical processes during combustion of intumescent fire-protective coatings// Combustion, Explosion and Shock Waves. 2001. T. 37, No. 2, C. 178-186

[6] Kuznetsov G.V., Mamontov G. Ya., Taratushkina G. V. Numerical simulation of ignition of a condensed substance by a particle heated to high temperatures// Combustion, Explosion and Shock Waves. 2004. T 40, No. 1, C. 70-76 